\title{
The truncation of the stellar disc of NGC 6504
}

\author{
E. Florido ${ }^{1,3}$, E. Battaner ${ }^{1,3}$, A. Zurita ${ }^{1,3}$, and A. Guijarro ${ }^{1,2,3}$ \\ 1 Departamento de Física Teórica y del Cosmos, Universidad de Granada, Spain \\ e-mail: estrella@ugr.es \\ 2 Centro Astronómico Hispano Alemán, Almería, Spain \\ 3 Instituto Carlos I. Física Teórica y Computacional, Universidad de Granada, Spain
}

Received 4 July 2007 / Accepted 19 July 2007

ABSTRACT

\begin{abstract}
Context. This is the first work reporting observations of the truncation of a stellar disc, in both the optical and the NIR spectral ranges. No galaxy has been observed at both wavelengths with the required depth. The optical radial profiles of spiral galaxy discs seem to suggest a double exponential behaviour, whilst NIR profiles seem to show a real truncation.

Aims. We study one galaxy with the best available resolution and detail, in both optical and NIR bands, with the same deprojection method and similar reduction procedures, to see if the different descriptions are real.

Methods. Optical observations ( $V$ band) were made with the William Herschel Telescope. NIR observations $\left(J, H, K_{\mathrm{s}}\right)$ were made with the CAIN infrared camera on the Carlos Sánchez Telescope in Tenerife (Spain). The deprojection method was that described in Florido et al. (2001, A\&A, 378, 82; 2006a, A\&A, 455, 467).

Results. NGC 6504 has a real truncation in both the optical and the NIR radial profiles. A double exponential does not fit the observed optical profile. The truncation radius is larger in the $V$ band than in the NIR by $\sim 10$ arcsec, about $3 \mathrm{kpc}$ (equivalent to about $10 \%$ ). We examine the constraints of this observation in theoretical scenarios.
\end{abstract}

Key words. galaxies: fundamental parameters - galaxies: photometry - galaxies: structure

\section{Introduction}

The truncation of stellar discs was discovered by van der Kruit (1979). In this and subsequent papers (van der Kruit \& Searle 1981a,b, 1982a,b) the truncation was described as a relatively sharp outer edge in the surface brightness distribution. Since then, there have been many interesting works (Barteldrees \& Dettmar 1994; de Grijs et al. 2001, and others) on disc truncations. In the pioneer papers by van der Kruit \& Searle, there was a truncation radius, $R_{\max }$, beyond which the surface brightness drops to undetectable values. The interpretation of these and later works was that the truncation was complete, i.e. that there was no emission beyond $R_{\max }$ (see, for instance, Wainscoat et al. 1992), and this is the actual meaning of the word "truncation". The truncation radius must therefore be estimated by extrapolation. The difference between the observed light profile and the extrapolated exponential of the inner disc was called the truncation curve by Florido et al. (2001, hereinafter F1), who suggested a $\left(R_{\max }-R\right)^{-\alpha}$ profile with $\alpha$ close to unity. The conclusions obtained by F1 and Florido et al. (2006a, hereinafter F6A) were based on NIR imaging of edge-on galaxies.

Recent optical observations have shown that there is no real truncation (as defined by van der Kruit), but a break separating the inner and the outer exponential surface brightness radial profiles, with an outer radial scale length which is usually, but not always, shorter than the inner one. This description has been proposed by Pohlen et al. (2002a, 2004), Pohlen \& Trujillo (2006), Erwin et al. (2005) and others. Pérez (2004) and Trujillo $\&$ Pohlen (2005) have shown that young galaxies at $z \sim 1$ can also be described with a double exponential. The break is noticeably sharp.
The question arises: are these real truncations or breaks? Are some stellar discs truncated and others simply "broken"? Are galaxy disc light profiles truncated in the NIR and "broken" in the optical? Are we dealing with the same phenomenon? The aim of this work is to answer these questions. To date, no galaxy has been measured in both the NIR and the optical, at least with the depth required for measuring the faint outer parts of galaxy discs. Another problem when comparing results is that some authors observe face-on galaxies and others edge-on ones, therefore using very different techniques, with the deprojecting method being particularly important. It would therefore be very interesting for one team to perform detailed observations of one single galaxy, with the highest quality of images in both the optical and the NIR, and with the same deprojection technique, thereby avoiding differences in reduction methods.

The selected galaxy was NGC 6504, an edge-on Sab galaxy with a total $B$ magnitude of 13.05 . The reason for this selection is that in F1 it was not clear if this galaxy was truncated or "broken"; no other selection criterion was taken into account. The deprojection technique was that presented in F1 and used in F1 and F6A. In both papers the error estimation introduced by this method was discussed in detail. It is worth mentioning here that the truncation curve is modified after deprojection, but the truncation radius is not $(\mathrm{F} 1)$.

\section{Observations}

The $V$ band imaging of NGC 6504 was obtained in service mode at the $4.2 \mathrm{~m}$ William Herschel Telescope (WHT) at the Observatorio del Roque de Los Muchachos in La Palma, using the prime focus imaging platform (PFIP) instrument and the 

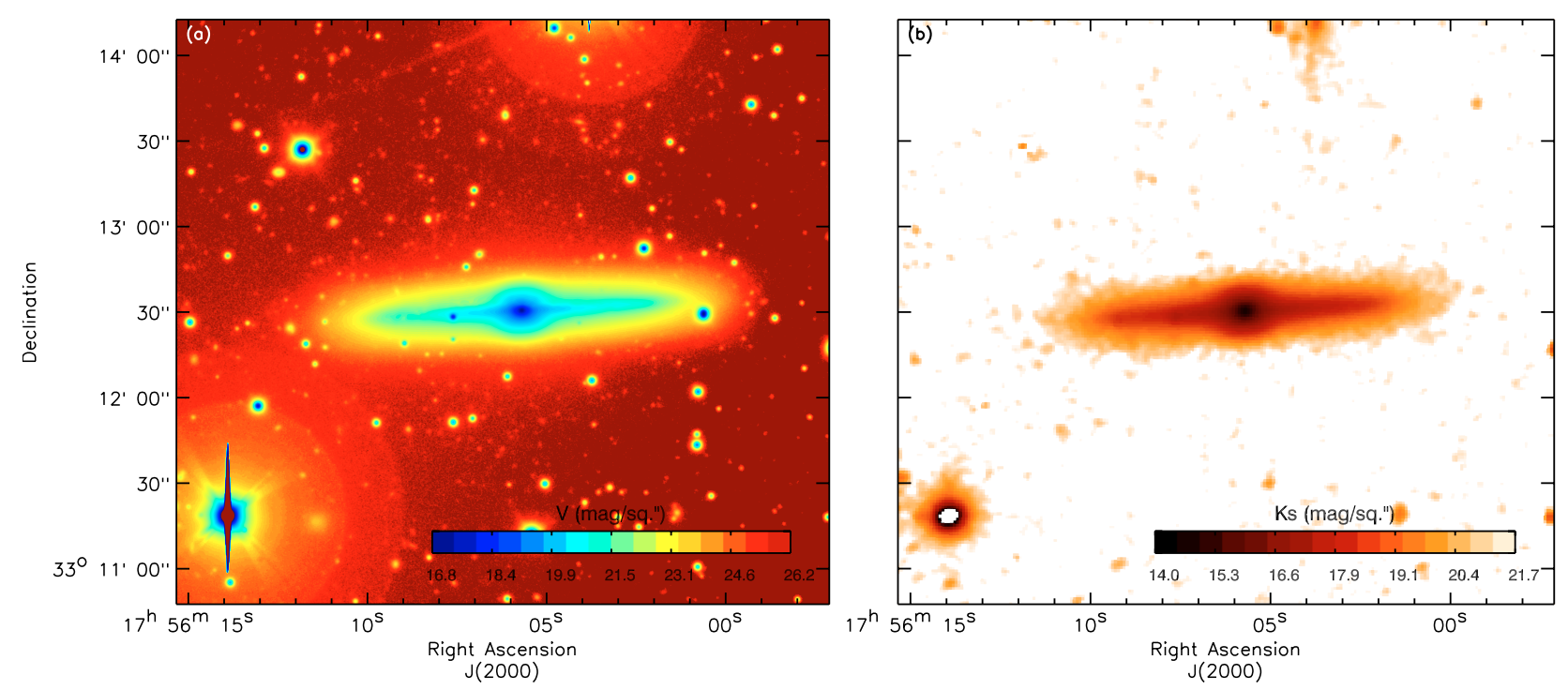

Fig. 1. a) $V$-band image of NGC 6504. b) $K_{\mathrm{s}}$-band image of NGC 6504 where the foreground stars have been removed for obtaining the surface brightness profiles (see Sect. 3 or F1 for further details). Both images show the same field of view.

Harris $V$ filter. The observations were taken on July 10, 2003 under photometric sky and $\sim 1^{\prime \prime}$ seeing. The PFIP detector is a mosaic of two thinned $2048 \times 4096 \mathrm{EEV}-42-80$ CCDs providing a field of view of $\sim 16.1^{\prime} \times 16.1^{\prime}$ and a pixel scale of $0.236^{\prime \prime} /$ pix. Given the relatively small angular size of the galaxy $\left(\sim 2.6^{\prime} \mathrm{di}-\right.$ ameter at $25 \mathrm{mag} / \operatorname{arcsec}^{2}$ ), it was centred on CCD2. NGC 6504 was observed for a total integration time of $6800 \mathrm{~s}$ (split into 42 images of $150 \mathrm{~s}$, one image of $200 \mathrm{~s}$ and one of $300 \mathrm{~s}$ ), during which the airmass varied between 1.06 and 1.95 .

Three different Landolt (Landolt 1992) standard star fields were observed during the night (on CCD2) at airmasses ranging from 1.08 to 2.6 .

The data were processed using standard techniques with IRAF $^{1}$. These comprise overscan subtraction and bias, and flat field corrects. The individual images were then sky substracted, aligned and combined. The spatial resolution of the final combined images is $1.1^{\prime \prime}$.

The IRAF package DAOPHOT was used to perform aperture photometry of the 11 stars of the Landolt standard star fields. The photometric calibration (which does not take into account colour terms) yielded a photometric zero point for ADU s ${ }^{-1}$ of $26.20+/-0.01$, and an extinction coefficient of $-0.109+/-0.005$. The limiting magnitude for signal-to-noise ratio per pixel equal to 3 in the final reduced image is $26.3 \mathrm{mag} \operatorname{arcsec}^{-2}$.

The image was astrometrically calibrated using the task ASTROM in GIPSY (van der Hulst et al. 1992), and identifying our foreground stars in the Palomar plates. The accuracy of the astrometric calibration was $\sim 0.3^{\prime \prime}$. The final reduced image is shown in Fig. 1a.

The NIR observations and data reduction techniques on which the results presented in this paper are based were described in detail in F1. These observations were carried out at the $1.5 \mathrm{~m}$ CST in the Teide Observatory, Tenerife, with the infrared camera CAIN.

The IRAF package was also used for the NIR bands. Flatfielding and sky substraction were performed very carefully

${ }^{1}$ IRAF is distributed by the National Optical Astronomy Observatories, which is operated by the Association of Universities for Research in Astronomy, Inc. (AURA) under cooperative agreement with the National Science Foundation. as we were dealing with extremely low brightness at the rim of the galaxy. To this end, the telescope was pointed alternately to six sky fields, also alternating object-sky, in order to correct properly for the fast atmospheric variations.

Star substraction was carried out following methods described in F1, following verification that this substraction was sufficient to obtain the truncation curve. Figure $1 \mathrm{~b}$ shows the star substracted $K_{\mathrm{S}}$ image. However, a bright star seen in the SE part of the galaxy in the $V$ band could have distorted the $V$-profile and was eliminated from the analysis. Calibration was performed using the 2MASS catalogue. After these processes, the galaxy was rotated to render the $x$-axis and the major axis coincident. Then we obtained profiles at several $z$ (distance from the galactic plane). By using the IMPLOT task the position of the $3 \sigma$-level was determined. We deprojected the $z$-profiles with the numerical method described in F1, and finally all the $z$-profiles were summed to obtain the final radial profile.

\section{Results}

In Figs. 2-4 we plot the NW side $V$-band profile compared with those in $J, H$ and $K_{\mathrm{s}}$ at both sides. All these curves are deprojected, and only points above $3 \sigma$ (before deprojection) are plotted. The $V$ curve has higher spatial resolution and reaches larger radii. We think there is no need of a statistical analysis to conclude directly from these figures that:

1. None of the profiles correspond to a double exponential description. In particular, the $V$ band profile shows a monotonic decrease, without a break. The slope becomes monotonically larger until reaching $V=28.5 \mathrm{mag} / \operatorname{arcsec}^{2}$ where the slope is extremely high. These profiles are interpreted as a non-sharp but complete truncation.

2. There is a clear difference between the truncation radius in the optical and the NIR bands. An extrapolation provides an estimated value of $R_{\max }(V)=85 \mathrm{arcsec}$ and $R_{\max }\left(K_{\mathrm{s}}\right)=74$ arcsec. This difference corresponds to $\approx 3.3 \mathrm{kpc}$, assuming a distance of $63.18 \mathrm{Mpc}$ for this galaxy. The difference between the NIR bands is not as high. A colour dependence of the truncation radius was already found by Florido et al. (2006b; thereafter F6B) where 


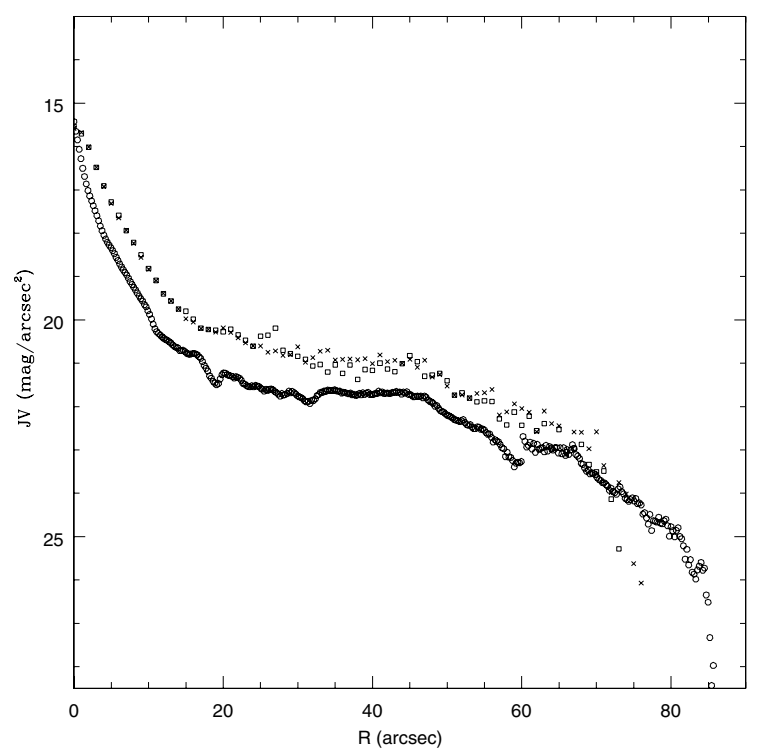

Fig. 2. $V$ band profile of NGC 6504 (circles) compared with the eastern side (squares) and the western side (crosses) profiles in $J$ band.

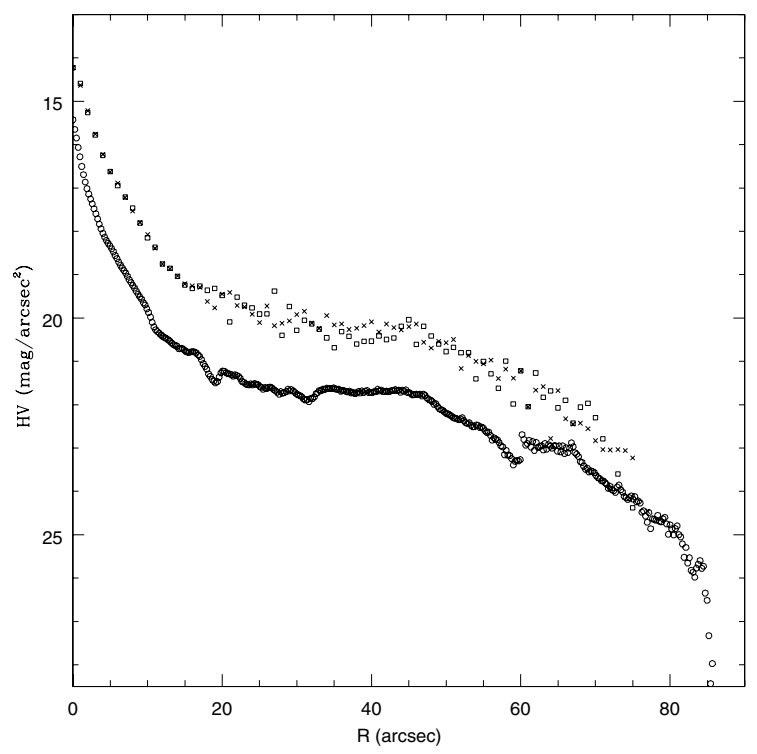

Fig. 3. $V$ band profile of NGC 6504 (circles) compared with the eastern side (squares) and the western side (crosses) profiles in $H$ band.

$R_{\max }(J)>R_{\max }\left(K_{\mathrm{S}}\right)$ for all galaxies in the sample, with only one exception, NGC 6504.

3 . Even if this conclusion cannot be reached with only one galaxy, this result strongly suggests that NIR truncations are associated with optical truncations. However, the location of the truncation radius depends on the colour. Nevertheless, the galaxy NGC 6504, and probably many others, does have a real truncation.

\section{Discussion}

We should now consider the kind of restraints the results of this paper introduce to the different proposed scenarios for explaining disc truncations. This discussion should take into account the arguments given by van der Kruit (2007) in a recent paper. We could classify these scenarios into small and large scale dynamics. In small scale dynamic models, the truncation or break

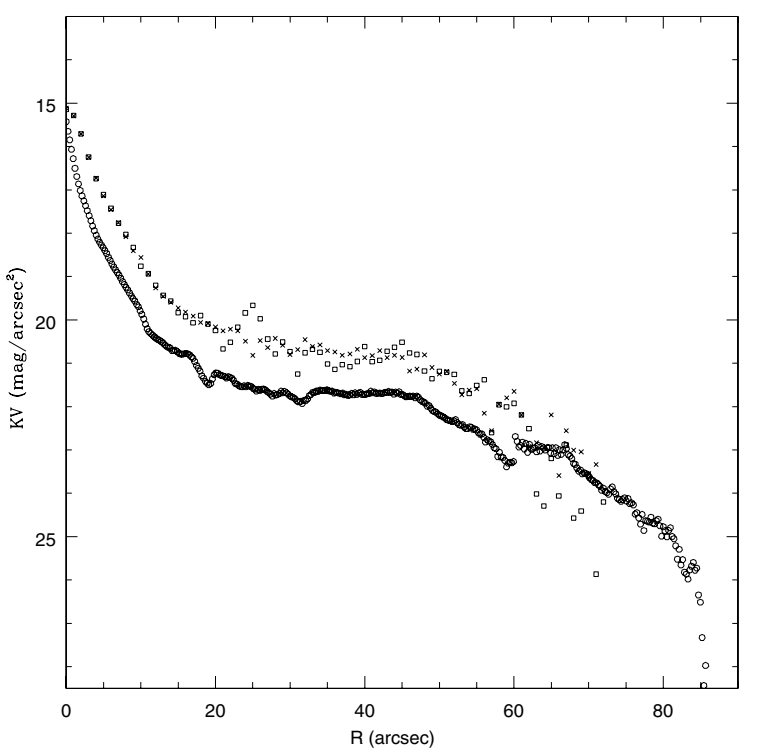

Fig. 4. $V$ band profile of NGC 6504 (circles) compared with the eastern side (squares) and the western side (crosses) profiles in $K_{\mathrm{s}}$ band.

is due to some inability of the gas to form stars, for instance, due to a threshold in the density (van der Kruit \& Searle 1981a; Kennicutt 1989; Schaye 2004), or due to an excessive local angular momentum in the collapsing clouds induced by differential rotation (Fall \& Efstathiou 1980).

Van der Kruit (2007) has provided abundant bibliographic and direct findings about a sudden drop in the rotation curve at the truncation radius. This drop indicates a sudden decrease of the total density (gas + stars) which, if confirmed, should be an important constraint to small scale dynamic models. Battaglia et al. (2006) also found a drop of $25 \mathrm{~km} \mathrm{~s}^{-1}$ in NGC 5055.

Van der Kruit (2007) proposes that the disc of the galaxy was formed in two steps. In the first step the inner disc was formed with a maximum size given by the truncation radius, and this boundary was set by the maximum specific angular momentum in the material forming the disc. The extragalactic gas falling in later, in the second step, produces the outer warped gaseous region.

This model implies in-falling motions, and is therefore considered as a large scale dynamic process. This scenario should take into account that optical warps exist in a region formed before the infalling, i.e. for $R<R_{\max }$. There are several catalogues of optical warps (Sánchez-Saavedra et al. 1990, 2003; Reshetnikov \& Combes 1999), and for $z \sim 1$ spirals (Reshetnikov et al. 2002). In addition, differential precession could destroy warps. We understand, however, that in the van der Kruit scenario, what is essential is that there was a first step in which $R_{\max }$ was established; the warps in the outer disc could be produced by other, or additional, means.

The magnetic model (Battaner et al. 2002) also involves large scale dynamical processes. Here, there is an inward magnetic force acting on the gas. When stars are formed this force no longer acts; therefore stars can move outwards and even escape. The truncation occurs at a point beyond which stars escape from the galaxy. It is noteworthy that, at least on quantitative grounds, this model is able to explain both truncations and "antitruncations". The word "antitruncation" was introduced by Erwin et al. (2005) for galaxies with the radial scale-length of the outer exponential larger than that of the inner one. In the NIR, antitruncations are found as well, NGC 2654 being a good example (F6B). 
In the magnetic model, the antitruncation should correspond to that region in which recently formed stars would have moved outwards but not escaped. Beyond this antitruncation region the truncation cut-off would exist. It should be noted here that the magnetic model is able to explain the correlation between $R_{\max }$ (or $R_{\text {break }}$ ) and the rotation velocity for larger $R$ found by Pohlen et al. (2004) and F6B.

The conclusion of this work is based only on one galaxy but it could be representative of a frequently observed type of galaxy. Real truncations in the NIR have been observed: NGC 4013, NGC 4217, NGC 5981 (F1), NGC 522, NGC 2862, NGC 3279 and MCG-01-05-047 (F6A) are good examples. The colour dependence was anticipated in F6B.

The model by van der Kruit (2007) and the magnetic model do explain the existence of a real truncation, but the colour dependence is somewhat puzzling. We have shown that the IR truncation (which traces older stars) takes place at smaller galactocentric radii than the optical. In the former model, the outer disc could have been growing with time and therefore the truncation radii could have been displaced to larger radii differentially in the optical. In the latter model, new born stars beyond $R_{\max }$ would be in the process of escaping, which could increase the optical truncation radius, as the escaping time can be of the order of $10^{8}$ years.

Acknowledgements. This paper has been supported by the "Consejería de Educación y Ciencia de la Junta de Andalucía" and by the "Secretaría de Estado de Política Científica y Tecnológica" (AYA2004-08251-C02-02,ESP200406870-C02-02). The WHT is operated on the island of La Palma by the Isaac Newton Group in the Spanish Observatorio del Roque de los Muchachos of the Instituto de Astrofísica de Canarias. We are very grateful to the ING staff for taking the service $V$ band observations. The CST is operated on the island of Tenerife by the Instituto de Astrofísica de Canarias.

\section{References}

Barteldrees, A., \& Dettmar, R. J. 1994, A\&AS, 103, 475

Battaglia, G., Fraternali, F., Oosterloo, T., \& Sanicisi, R. 2006, A\&A, 447, 49

Battaner, E., Florido, E., \& Jiménez-Vicente, J. 2002, A\&A, 388, 213

Erwin, P., Beckman, J. E., \& Pohlen, M. 2005, ApJ, 626, L81

Fall, S. M., \& Efstathiou, G. 1980, MNRAS, 193, 189

Florido, E., Battaner, E., Guijarro, A., Garzón, F., \& Jiménez-Vicente, J. 2001, A\&A, 378, 82 (F1)

Florido, E., Battaner, E., Guijarro, A., Garzón, F., \& Castillo-Morales, A. 2006a, A\&A, 455, 467 (F6A)

Florido, E., Battaner, E., Guijarro, A., Garzón, F., \& Castillo-Morales, A. 2006b, A\&A, 455, 475 (F6B)

de Grijs, R., Kregel, M., \& Wesson, K. H. 2001, MNRAS, 324, 1074

van der Hulst, J. M., Terlouw, J. P., Begeman, K., Zwitser, W., \& Roelfsema, P. R. 1992, in Astronomical Data Analysis Software and Systems I, ed. D. M. Worall, C. Biemesderfer, \& J. Barnes, ASP Conf. Ser., 25, 131

Kennicutt, R. C. 1989, ApJ, 344, 685

van der Kruit, P. C. 1979, A\&AS, 38, 15

van der Kruit, P. C. 2007 [arXiv: astro-ph/0702486]

van der Kruit, P. C., \& Searle, L. 1981a, A\&A, 95, 105

van der Kruit, P. C., \& Searle, L. 1981b, A\&A, 95, 116

van der Kruit, P. C., \& Searle, L. 1982a, A\&A, 110, 61

van der Kruit, P. C., \& Searle, L. 1982b, A\&A, 110, 79

Landolt, A. U. 1992, AJ, 104, 340

Pérez, I. 2004, A\&A, 427, L17

Pohlen, M., \& Trujillo, I. 2006, A\&A, 454, 759

Reshetnikov, V., \& Combes, F. 1999, A\&A, 138, 131

Reshetnikov, V., Battaner, E., Combes, F., \& Jiménez-Vicente, J. 2002, A\&A, 382,513

Sánchez-Saavedra, M. L., Battaner, E., \& Florido, E. 1990, MNRAS, 246, 458

Sánchez-Saavedra, M. L., Battaner, E., Guijarro, A., López-Corredoira, M., \& Castro-Rodríguez, N. 2003, A\&A, 399, 457

Schaye, J. 2004, ApJ, 609, 667

Trujillo, I., \& Pohlen, M. 2005, ApJ, 630, L17

Wainscoat, R., Cohen, M., Völk, Walker, H. J., \& Schwartz, D. E. 1992, ApJS, 83,111 